\title{
Comparison of dose volume parameters evaluated using three forward planning - optimization techniques in cervical cancer brachytherapy involving two applicators
}

\author{
Bikramjit Chakrabarti, MD, DNB, Somapriya Basu-Roy, MSc, PhD, Sanjay Kumar Kar, MSc, Sounik Das, MBBS, \\ Annesha Lahiri, MBBS \\ Department of Radiotherapy, Institute of Post-Graduate Medical Education and Research and Seth Sukhlal Kernani Memorial Hospital, \\ Kolkata, India
}

\begin{abstract}
Purpose: This study is intended to compare dose-volume parameters evaluated using different forward planningoptimization techniques, involving two applicator systems in intracavitary brachytherapy for cervical cancer. It looks for the best applicator-optimization combination to fulfill recommended dose-volume objectives in different high-doserate (HDR) fractionation schedules.

Material and methods: We used tandem-ring and Fletcher-style tandem-ovoid applicator in same patients in two fractions of brachytherapy. Six plans were generated for each patient utilizing 3 forward optimization techniques for each applicator used: equal dwell weight/times ('no optimization'), 'manual dwell weight/times', and 'graphical'. Plans were normalized to left point $\mathrm{A}$ and dose of $8 \mathrm{~Gy}$ was prescribed. Dose volume and dose point parameters were compared.

Results: Without graphical optimization, maximum width and thickness of volume enclosed by $100 \%$ isodose line, dose to $90 \%$, and $100 \%$ of clinical target volume (CTV); minimum, maximum, median, and average dose to both rectum and bladder are significantly higher with Fletcher applicator. Even if it is done, dose to both points B, minimum dose to $\mathrm{CTV}$, and treatment time; dose to $2 \mathrm{cc}\left(\mathrm{D}_{2 \mathrm{cc}}\right)$ rectum and rectal point etc.; $\mathrm{D}_{2 \mathrm{cc}}$, minimum, maximum, median, and average dose to sigmoid colon; $\mathrm{D}_{2 \mathrm{cc}}$ of bladder remain significantly higher with this applicator. Dose to bladder point is similar $(p>0.05)$ between two applicators, after all optimization techniques.

Conclusions: Fletcher applicator generates higher dose to both CTV and organs at risk (2 cc volumes) after all optimization techniques. Dose restriction to rectum is possible using graphical optimization only during selected HDR fractionation schedules. Bladder always receives dose higher than recommended, and 2 cc sigmoid colon always gets permissible dose. Contrarily, graphical optimization with ring applicators fulfills all dose volume objectives in all HDR fractionations practiced.
\end{abstract}

Key words: cervical cancer, cervix, carcinoma, dosimetry, optimization.

\section{Purpose}

Cervical cancer is the fourth most common cancer in women worldwide [1]. In 2012, there were an estimated 266,000 deaths from cervical cancer worldwide, accounting for $7.5 \%$ of all female cancer deaths [2]. A large majority (around $85 \%$ ) of the global burden occurs in the less developed regions, where it accounts for almost $12 \%$ of all female cancers. Almost nine out of ten $(87 \%)$ cervical cancer deaths occur in the less developed regions [2]. Except for the very early cases, radiation therapy is the major curative treatment option for this disease. Brachytherapy plays an anchor role in management of cervical cancer and forms an integral part of radiation therapy. Intracavitary brachytherapy remains the most commonly practiced form of brachytherapy for cervical cancer [3].

While various recommendations are available for intracavitary insertion techniques, dosage schedule, dose prescriptions, and reporting of the procedure, to date, no guideline is available regarding the selection of the applicator type and optimization technique. A choice of appli- 
cator is rather arbitrary [4], and depends on availability, patients' pelvic geometry as well as extent of disease. However, since the dose distribution in brachytherapy follows inverse square law, different patterns of dose distribution were generated with different applicator systems, some of these may help to achieve higher therapeutic ratio by sparing the organs at risk and, at the same time, covering the target in a more satisfactory manner than the other.

\section{Objectives of our study}

The recommended dose volume objective for clinical target volume (CTV) is to deliver a minimum of $100 \%$ of prescribed dose to $90 \%$ volume $\left(\mathrm{D}_{90}\right)$ of CTV. Whereas, the dose volume constraints recommended for different organs at risk (OARs) are: (a) less than 75 Gy two-gray equivalent dose $\left(\mathrm{EQD}_{2}\right)$ to $2 \mathrm{cc}\left(\mathrm{D}_{2 \mathrm{cc}}\right)$ volume of contoured rectum and sigmoid colon, and (b) less than $90 \mathrm{~Gy} \mathrm{EQD}_{2}$ to 2 cc volume of urinary bladder [5,6,7]. Different highdose-rate (HDR) brachytherapy fractionation protocols are currently practiced after external beam radiotherapy (EBRT). To engender total $\mathrm{EQD}_{2}$ to the contoured volumes, different fractionation protocols will have different permissible $\mathrm{D}_{2 \mathrm{cc}}$ for OARs per fraction of brachytherapy. Our study aims to compare dose volume and dose point parameters using three-dimensional (3D) treatment planning for two different applicator systems and three forward planning-optimization techniques in same patients, to find out the best suitable applicator-optimization combination to fulfill dose volume objectives. The two applicator systems are tandem-ring and Fletcher-style tandem ovoid applicators. The three optimization techniques are 'no optimization', a term used in our treatment planning software, which sets equal dwell weight/times of all dwell positions; 'manual optimization' setting weight of ovoid/ ring dwell positions to 0.33 of those in tandem, as proposed by a study by Obed et al. [8]; and 'graphical' to drag the isodose lines to conform to the contoured CTV and sparing the OARs.

\section{Material and methods}

\section{Patients selection}

At our institution, we treat nearly 300 newly-diagnosed cases of cervical cancer every year. Among them, 22 patients (Table 1) suffering from locally advanced disease (stage IIA-IIIB), squamous cell carcinoma in histology with good performance status (ECOG 0-2) and no other significant co-morbidities (which might alter treatment outcomes) were selected for this study. Patients with gross residual tumor after EBRT or having distort-

Table 1. Patient characteristics according to stage

\begin{tabular}{lcc} 
Stage of the disease & Number of patients & $\%$ \\
\hline IIA & 5 & 22.7 \\
\hline IIB & 11 & 50 \\
\hline IIIB & 6 & 27.3
\end{tabular}

ed anatomy of vaginal fornices were excluded from the study.

\section{External beam radiotherapy technique}

All patients received a dose of 50 Gy to the whole pelvis in conventional fractionation with four field box technique prior to brachytherapy, along with concurrent chemotherapy of cisplatin $40 \mathrm{mg} / \mathrm{m}^{2}$ given weekly. In patients with extensive parametrial disease involvement, a further 10 Gy parametrial boost in conventional fractionation was delivered by parallel opposed antero-posterior beams. However, in these patients, brachytherapy insertions were intertwined between the parametrial boost fractions to keep the total duration of treatment within eight weeks. When interdigitating brachytherapy, caution was taken not to administer weekly chemotherapy on a brachytherapy day, but to deliver rather on an EBRT day to avoid the possibility of increased adverse effects due to normal-tissue sensitization.

\section{Brachytherapy insertion}

Oral anxiolytic and stool softener were given to the patient the night before the procedure. Intracavitary insertion was done under intravenous sedation by an experienced radiation oncologist. Tandem-ring and the Fletcher-style tandem ovoid applicators (Nucletron, an Elekta company, Stockholm, Sweden) were used for same patients in two different insertions. For each patient included in this study, the type of applicators and sequence of insertion were randomly assigned using a blind envelope method. Patients were randomly assigned to receive first insertion using either ring or Fletcher applicator; the other type being used for the second insertion. This random assignment of the insertion sequence ensured that changes in local anatomy due to progressive tumor shrinkage, as well as general patient condition at the time of insertion would be balanced between uses of two applicator systems. To engender comparability, the selected basic parameters for insertion according to patient's utero-vaginal anatomy, e.g. the intra-uterine tandem length, angulation, and lateral separation were kept as similar as feasible for both applicators. Ovoid separation or ring diameter used were both the same, as permitting by patient's anatomy. All patients underwent a computerized tomographic (CT) scan (3 mm slice) using Philips Brilliance CT simulator (Philips Healthcare, Inc., Andover, MA, USA) after every insertion with the applicators in position.

\section{Brachytherapy contouring}

Oncentra ${ }^{\circledR}$ Brachy version 4.5.2 treatment planning system (Nucletron, an Elekta company, Stockholm, Sweden) was used for 3D treatment planning. Whole of the cervix with any parametrial extension was contoured following the guideline of American Brachytherapy Society (ABS) Cervical Cancer Brachytherapy Task group [6], extending superiorly up to the location where the uterus began to enlarge. If these could not be identified, a height of $3 \mathrm{~cm}$ was contoured for the cervix. A portion of tandem 
inside the cervix was included in the contoured clinical target volume (CTV). The whole of urinary bladder inclusive of the Foley's catheter bulb, whole of rectum, and sigmoid colon were contoured as organs at risk. ICRU reference points, namely point $\mathrm{A}$ and $\mathrm{B}$ on right and left sides, bladder, and rectal points were identified.

\section{Optimization techniques}

After catheter reconstruction, activation of dwell positions was done in similar fashion between two applicator systems to ensure comparability. Three types of optimization techniques, as described earlier, were carried out after each of two insertions by an experienced medical physicist and thus, six plans were created and saved for each patient for dosimetric comparison. All plans were normalized to left sided point A to ensure uniformity prior to comparison, while normalization was ascertained again after optimization. Three other optimization techniques, namely IPSA (inverse planning simulated annealing), HIPO (hybrid inverse planning optimization), and geometric optimization were not used as the first two are not licensed to our planning system and the last was not applicable. The usual practice at our department is prescription of $8 \mathrm{~Gy}$ to the normalized point A. A dose of 86 Gy $\mathrm{EQD}_{2}(\alpha / \beta=10)$ was delivered to point A. Prescription was reviewed by treating oncologist and radiation safety was checked by the radiation safety officer.

The procedure of brachytherapy insertion includes the use of intravenous sedation, CT imaging, and prescription of dose to point $A$, which were all in accordance with 'modifications from American Brachytherapy Society (ABS) 2012 consensus guidelines for practice in resource-limited settings (RLSs)' [9]. Treatment was effected using ${ }^{192}$ Ir source in HDR-microselectron brachytherapy set-up (Nucletron, an Elekta company, Stockholm, Sweden).

\section{Documentation}

All dose volume parameters $\mathrm{D}_{90}, \mathrm{D}_{100}, \mathrm{~V}_{100}$ (volume covered by $100 \%$ isodose), $\mathrm{V}_{150}$ (volume covered by $150 \%$ isodose), and $\mathrm{V}_{200}$ (volume covered by $200 \%$ isodose) for CTV and $\mathrm{D}_{0.1 \mathrm{cc}}$ (dose to 0.1 cc volume), $\mathrm{D}_{1 \mathrm{cc}}$ (dose to $1 \mathrm{cc}$ volume), $\mathrm{D}_{2 \mathrm{cc}}, \mathrm{V}_{20}$ (volume covered by $20 \%$ isodose), $\mathrm{V}_{50}$ (volume covered by $50 \%$ isodose), $\mathrm{V}_{85}$ (volume covered by $85 \%$ isodose), and $\mathrm{V}_{95}$ (volume covered by $95 \%$ isodose) for rectum, sigmoid colon, and urinary bladder were documented. Dose point parameters like dose to bladder and rectal point, contralateral un-normalized point A and bilateral point B were noted. Additionally, width (the maximum and at the level of point $\mathrm{A}$ ) and maximum height of volume enclosed by $100 \%$ isodose curve, total reference air kerma (TRAK) at $1 \mathrm{~m}$, and treatment time were recorded.

\section{Statistical analysis}

Online calculator available at http:/ / www.graphpad. com was used for data analysis. Paired $t$-test was used to compare dose-volume, dose-point, and other parameters between two applicator systems using each of three optimization techniques. $\mathrm{EQD}_{2}$ calculations had been done using LQ (linear quadratic) spreadsheet available at American Brachytherapy Society's http://www.americanbrachytherapy.org/guidelines/LQ_spreadsheet.xls website.

\section{Discussion}

\section{Treatment modalities}

American Brachytherapy Society (ABS) recommends that EBRT and brachytherapy should be completed in less than 8 weeks with interdigitating these if needed for better local tumor control and survival. When interdigitated, chemotherapy should be administered on an EBRT day and not on a brachytherapy day to avoid potential for increased complications [5]. Superior dosimetric coverage of parametrium has been reported after addition of intensity modulated radiotherapy (IMRT) [10] or interstitial brachytherapy (ISBT) [11] with intracavitary brachytherapy.

\section{Imaging}

The Groupe Européen de Curiethérapie - European Society for Radiotherapy \& Oncology (GEC-ESTRO) recommends the use of magnetic resonance imaging (MRI) with $\mathrm{T} 2 \mathrm{w}$ pulse sequences both at the time of diagnosis and brachytherapy [12], since there is significant reductions in normal tissue toxicity, improvements in local control, and overall survival after MRI-guided adaptive brachytherapy [13]. Although magnetic resonance imaging (MRI) is superior to CT for the differentiation of gross tumor volume (GTV) and parametrial disease [14], CT and MRI provide basically similar quality for discrimination of bladder, rectum, sigmoid, bowel, and vagina [7]. In limited resource setting, MRI based preplanning at the first brachytherapy application and consecutive CT/ MRI data fusion has been demonstrated to be safe and feasible by Dolezel et al. $[15,16]$. In absence of MRI, CT scans alone when used for brachytherapy planning can ensure organs at risk (OAR) doses to be kept within acceptable limits. However, CT based target volumes have been overestimated as compared to MRI volumes $[15,17]$.

\section{Contouring}

For locally advanced disease, point A based prescription can result in under dosage or geographic misses as has been documented on CT images $[15,18,19]$. Imageguided brachytherapy with $3 \mathrm{D}$ volume contouring and assessment of dose volume parameters should be done to ensure good coverage to target and sparing of organs at risk. For institutions that utilize $\mathrm{CT}$, the width of the cervix and any parametrial extension should be contoured as CTV-CT [6]. The superior border of the cervix should extend at the location where the uterus begins to enlarge or at least $1 \mathrm{~cm}$ above either the uterine vessels identified by IV contrast. If these cannot be identified, a height of $3 \mathrm{~cm}$ should be contoured for the cervix [6]. When reporting DVH (dose volume histogram) parameters, it should be always mentioned if the applicator is included in the 
contoured CTV or not [7]. For organ wall volumes up to $2-3 \mathrm{~cm}^{3}$, organ and organ wall contouring lead to almost identical numerical results [20], which allows for organ contouring only. If larger organ wall volumes are considered, organ wall contouring has to be performed [7].

\section{Dose prescription and optimization}

Dosimetry should be performed every time applicators are inserted to assess dose to the target volumes as well as the normal tissues, even if fixed geometry applicators are used [6]. Dose has been prescribed historically as milligram per hour of radium or total reference air kerma (TRAK), and is currently mainly prescribed to specific well-defined points (e.g. point A) or volumes [6]. Optimization in the 2000 ABS Guidelines [5] refers to setting lateral dose points adjacent to the applicator, based on radiographic localization. With 3D-imaging, optimization refers to starting with a customary loading of the full length of the tandem and the vaginal applicator (ovoids, ring, or cylinder), then modifying the dwell positions to reduce the dose to the OAR and ensure maximal tumor coverage. American Brachytherapy Society recommends that, although point $A$ dose should be recorded; the priority is to administer planned $\mathrm{EQD}_{2}$ to $\mathrm{D}_{90}$ (volume covered by $90 \%$ of the prescribed dose) of contoured clinical target volume (CTV) [5]. If prescription is based on a target volume and not on point $\mathrm{A}$, it is still possible to normalize to point $A$ by changing the normalization value until the prescribed isodose reaches a certain dimension [7].

\section{Assessment of dose volume parameters}

Cumulative dose volume histograms (DVH) are recommended for evaluation of the complex dose heterogeneity. DVH parameters for target volume are the minimum dose delivered to $90 \%\left(D_{90}\right)$ and $100 \%\left(D_{100}\right)$. Due to the steep dose gradient, small spikes in the contour cause large deviations in $D_{100}$. $D_{90}$ is less sensitive to these influences, and is therefore considered to be a more 'stable' parameter [7]. Volume enclosed by $150 \%\left(\mathrm{~V}_{150}\right)$ or $200 \%$ $\left(\mathrm{V}_{200}\right)$ of the prescribed dose is recommended for overall assessment of high-dose regions. Volume enclosed by $100 \%$ of prescribed dose $\left(\mathrm{V}_{100}\right)$ is recommended for quality assessment, only within a given treatment schedule. For OAR, the minimum dose to the most irradiated tissue volume recommended for reporting are $0.1,1$, and $2 \mathrm{~cm}^{3}$; optionally 5 and $10 \mathrm{~cm}^{3}$ [7].

\section{Optimum organ at risk volume}

Optimum bladder volume is essential to minimize dose to pelvic organs at risk. A study by Siavashpour [21] revealed that choosing a bladder with a volume of about $70 \mathrm{~cm}^{3}$ or less was recommended when taking into account the high-dose volume parameters for bladder, rectum, and sigmoid. Another study by Patra et al. [22] revealed that with distension of bladder, mean bladder dose and small volume dose parameters $(0.1 \mathrm{cc}, 1 \mathrm{cc}$, and $2 \mathrm{cc}$ ) of small intestine decreases. However, there is no significant change in these parameters of bladder, rectum, and sigmoid colon.

\section{Recommended prescribed dose}

$\mathrm{D}_{90}$ of contoured target volume should be equal to $100 \%$ of prescription dose. American Brachytherapy Society recommends $\mathrm{EQD}_{2}$ of $\geq 80$ Gy for patients with either a complete or a partial response with residual disease less than $4 \mathrm{~cm}$, and an $\mathrm{EQD}_{2}$ of 85-90 Gy for non-responders or those with tumors larger than $4 \mathrm{~cm}$ at the time of brachytherapy [5]. $D_{2 c c}$ of rectum or sigmoid less than $75 \mathrm{~Gy}$ and $D_{2 c c}$ of urinary bladder less than $95 \mathrm{~Gy}$ have been reported [6]. ABS recommends $\mathrm{EQD}_{2}$ limit for the rectum and sigmoid as 70-75 Gy and for the bladder as 90 Gy [5]. European study on MRI-guided brachytherapy in locally advanced cervical cancer (EMBRACE) [23] and Cancer Care Ontario (CCO) [24] also set same doses for organs at risk.

\section{Uncertainties}

Multiple applications can lead to a variation in the spatial position of applicators in relation to the pelvic organs, bones, and the organs at risk, mainly attributed to patient movement and tumor regression during the interval between multiple fractions [15]. These have been reported in terms of changes in the uterine axis, uterine length, colpostat separation, and vaginal packing or slippage of tandem, filling state of bladder and rectum etc. $[15,25]$. Thus, for accurate assessment of dose volume parameters, imaging after each insertion of brachytherapy is preferable. However, intra-fraction errors due to bowel movements or movement of patient cannot be ruled out. GEC-ESTRO (The Groupe Européen de Curiethérapie European Society for Radiotherapy and Oncology) made assumptions of full dose of external beam therapy in the volume of interest, identical location during fractionated brachytherapy, and contiguous volumes [7] to ignore these uncertainties.

\section{Comparison of two applicator systems}

Both ring and Fletcher applicators are based on the Manchester system, but their geometry is quite different (see Figure 1). Fletcher applicator system consists of three applicators: the intrauterine tandem, activation of source dwell positions along, which generates the dose longitudinally, and the two angulated lateral ovoids responsible for widening the dose laterally to point $\mathrm{A}$ and also antero-posteriorly. It has flexible geometry as the separation and the relative longitudinal position of the applicators can be adjusted. Although the three applicators are fixed by screws outside vagina, the positioning of the ovoids within the fornix may be asymmetric, as it largely depends on the shape and symmetry of the vaginal fornix. The assembled Fletcher apparatus exhibits a wider distribution at the level of the ovoids as well as thicker anterior/posterior distribution. The ring applicator system is a fixed-geometry two-applicator system, where the tandem and the single ring applicator can be fixed only at a particular slot inside the vagina, and operating oncologist is constrained to select one of the ring sizes available in his institution. Patients with shallow vaginal fornices or those having partially fixed utero-vaginal anatomy not matching the geometry of the available ring applicators, 

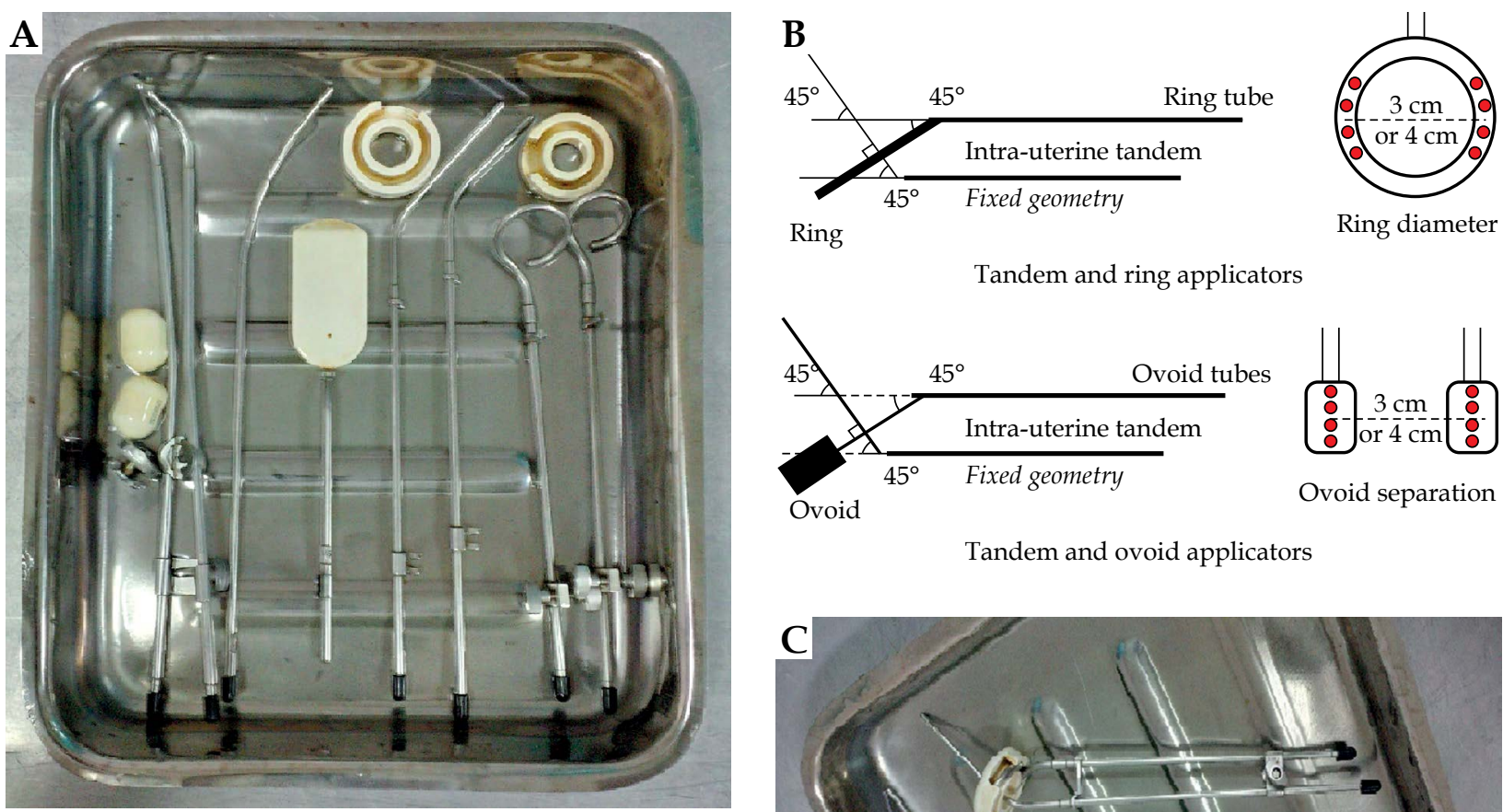

Tandem and ring applicators
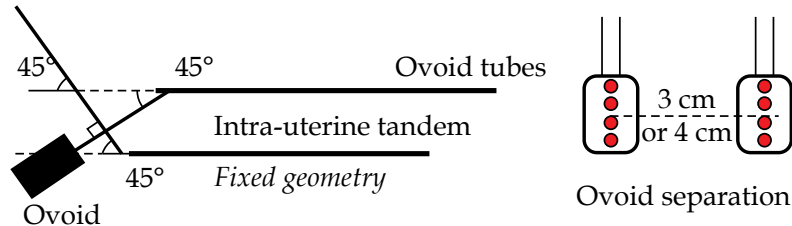

Ovoid separation

Tandem and ovoid applicators

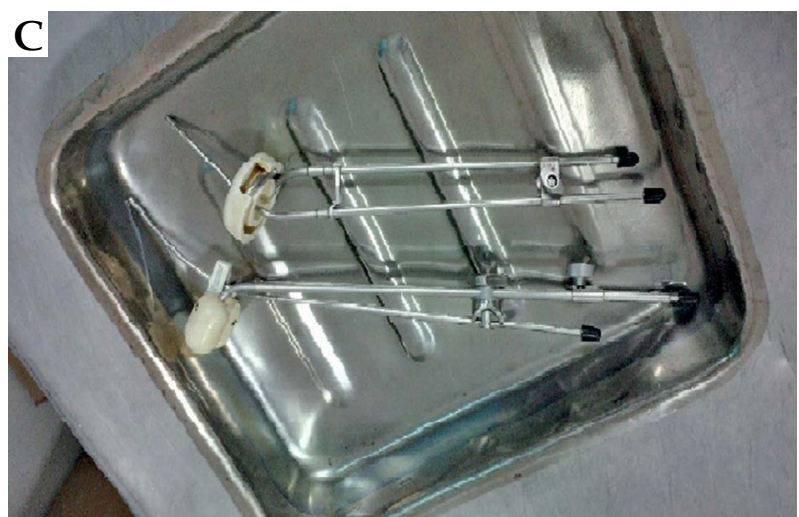

Fig. 1. Figure showing (A) intracavitary brachytherapy applicators: Fletcher applicator on the left and ring applicator on the right, (B) diagram showing comparative geometry of the two applicators, (C) assembled applicators - ring applicator above and Fletcher applicator below. Fletcher applicator is a flexible geometry three-applicator system, the positioning which may be asymmetric depending on the shape and symmetry of the vaginal fornix. The assembled Fletcher apparatus exhibits a wider distribution at the level of the ovoids as well as thicker anterior-posterior distribution. Ring applicator is a fixed-geometry two-applicator system. Patients with shallow vaginal fornices or partially fixed utero-vaginal anatomy not matching the geometry of the ring applicator cause difficulty in proper placement of the applicators

cause difficulty in proper placement of the applicators. However, if patient's anatomy is symmetric and favorable, some oncologists find insertion of ring applicator easier than Fletcher applicator.

\section{Trials comparing two applicator systems}

Dose distributions for Fletcher-style tandem ovoid (TO) and tandem-ring (TR) applicators have been compared at ICRU (International Commission on Radiation Units and Measurements) point A, bladder and rectal points using Abacus software in a study by Rehman et al. They observed that dose at point A was significantly higher and doses to bladder and rectal point were smaller (statistically insignificant) for the Fletcher applicators. They concluded that Fletcher applicator achieved a better dose distribution, thus predicting a better treatment outcome [26]. In a study by Levin et al. [27], dosimetric comparisons of these two applicators were investigated using CT-guided volume determination. The authors showed that even though the optimization point doses were similar between the two applicators, tandem-ovoid applicator clearly exhibited a larger isodose volume in comparison to ring applicator. A study conducted at University of Mississippi by Ma et al. [28] compared the short-term toxicity and dosimetry of these applicators. They observed that although the rectal $D_{2 c c}$ was statistically similar between TO and TR, the mean rectal dose in TR was lower. $\mathrm{V}_{95}, \mathrm{~V}_{85}, \mathrm{~V}_{50}$, and $\mathrm{V}_{20}$ were all significantly higher for TO than for TR $(p<0.018)$. Despite the larger isodose volume seen in TO, the percent of the CTV that received $100 \%$ of the prescription dose $\left(\mathrm{CTV}_{100 \%}\right)$ and the percent of prescription dose covering $90 \%$ of the CTV $\left(D_{90}\right)$ were not statistically different. This study had certain limitations. In order to modify the isodose curve to bring OAR $\mathrm{D}_{2 \mathrm{cc}}$ under GEC-ESTRO recommendations, optimization was achieved by adjusting the radioactive source loading patterns, dwell positions, and dwell times. Thus, the difference in dose distribution may had been offset by intentional optimization to spare organs at risk. For this reason, both sets of patients had similar short term toxicities. Furthermore, CTV was contoured retrospectively, and the two types of applicators were inserted by two surgeons in different patients. 


\section{Trials comparing different loading patterns}

An orthogonal imaging based study in Nigeria by Obed et al. [8] compared two loading patterns using only ring applicator system. They observed that the means of all evaluated dose parameters decreased when tandem-ring dwell time ratio $1: 1$ was modified to other dwell weightings, $1: 1-3: 1$. These reductions were $13.43 \%$ for ICRU volume in $\mathrm{cm}^{3}\left(\mathrm{ICRU}_{\mathrm{H}} \times \mathrm{ICRU}_{\mathrm{W}} \times \mathrm{ICRU}_{\mathrm{T}}\right), 9.83 \%$ for rectal dose, $6.68 \%$ for point B dose, $6.08 \%$ for treatment time, $5.90 \%$ for TRAK, and $1.08 \%$ for bladder dose [8]. No study to date has compared the effects of different optimization techniques or loading patterns along with different applicator systems in same patients.
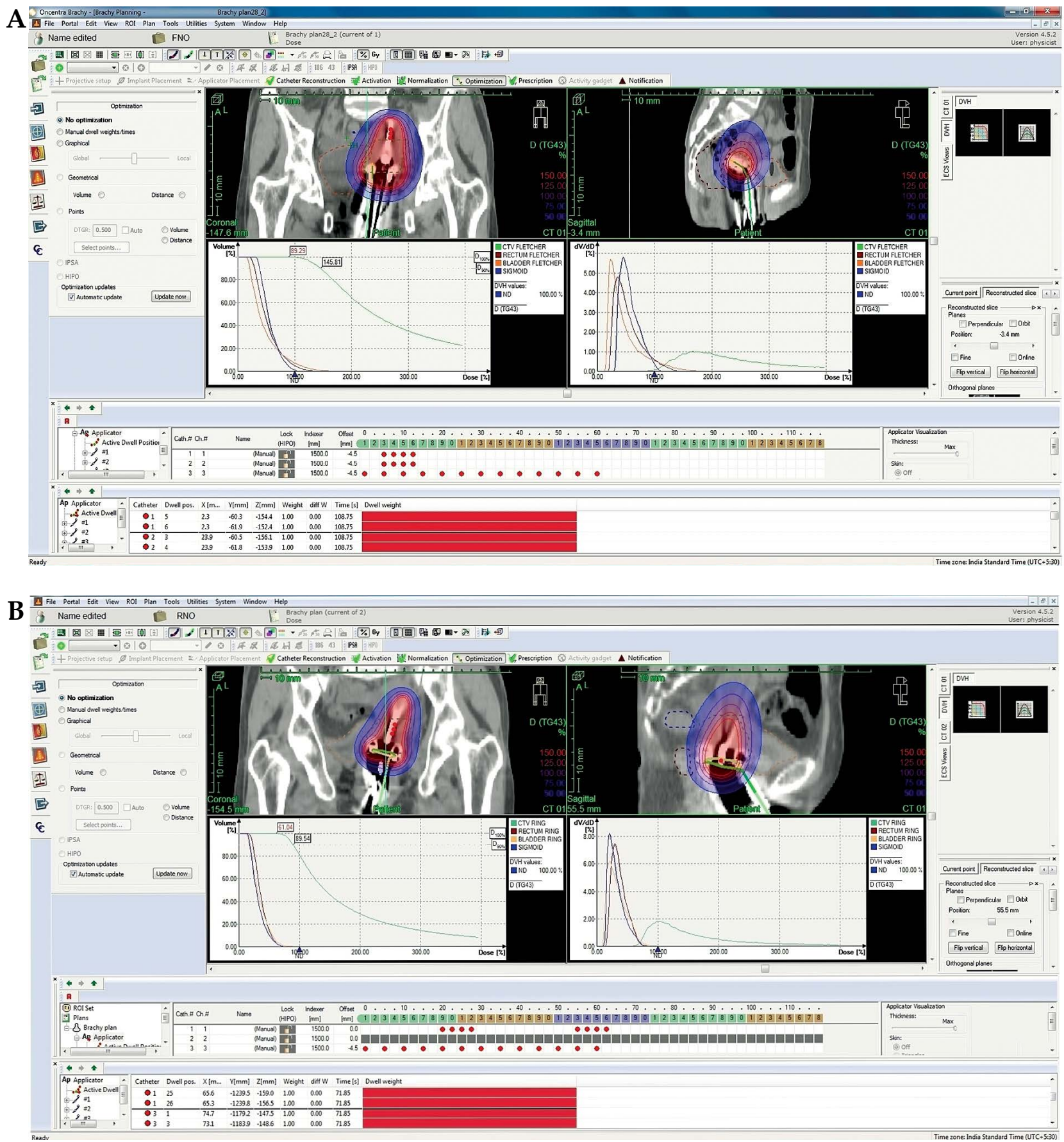

Fig. 2. A) Screenshots showing loading patterns, optimization techniques, isodose volumes, and cumulative and differential dose volume histogram generated after 'no optimization' using Fletcher tandem ovoid applicator. B) Although the loading patterns and optimization techniques are similar to Fletcher applicator as in Figure 2A, the 100\% isodose is narrower and thinner, and dose delivered to contoured volumes are lower after using ring applicator 


\section{Achieved results from our study and their implications}

Dose distribution from Fletcher applicator is both wider side to side and thicker antero-posteriorly even if the lateral separation of ovoids are identical with ring diameter and source loading patterns are planned in a sim- ilar way (see Figure 2). Unless graphical optimization is performed, maximum width and thickness of $100 \%$ isodose, TRAK at $1 \mathrm{~m}$ (Table 3), $\mathrm{V}_{100}, \mathrm{~V}_{150}, \mathrm{~V}_{200}$, and $\mathrm{D}_{90}, \mathrm{D}_{100}$ of CTV (Table 2) are statistically significantly $(p<0.0001)$ high with Fletcher applicator. This difference is nullified with graphical optimization. However, even if this is done, dose to un-normalized point A (on patient's
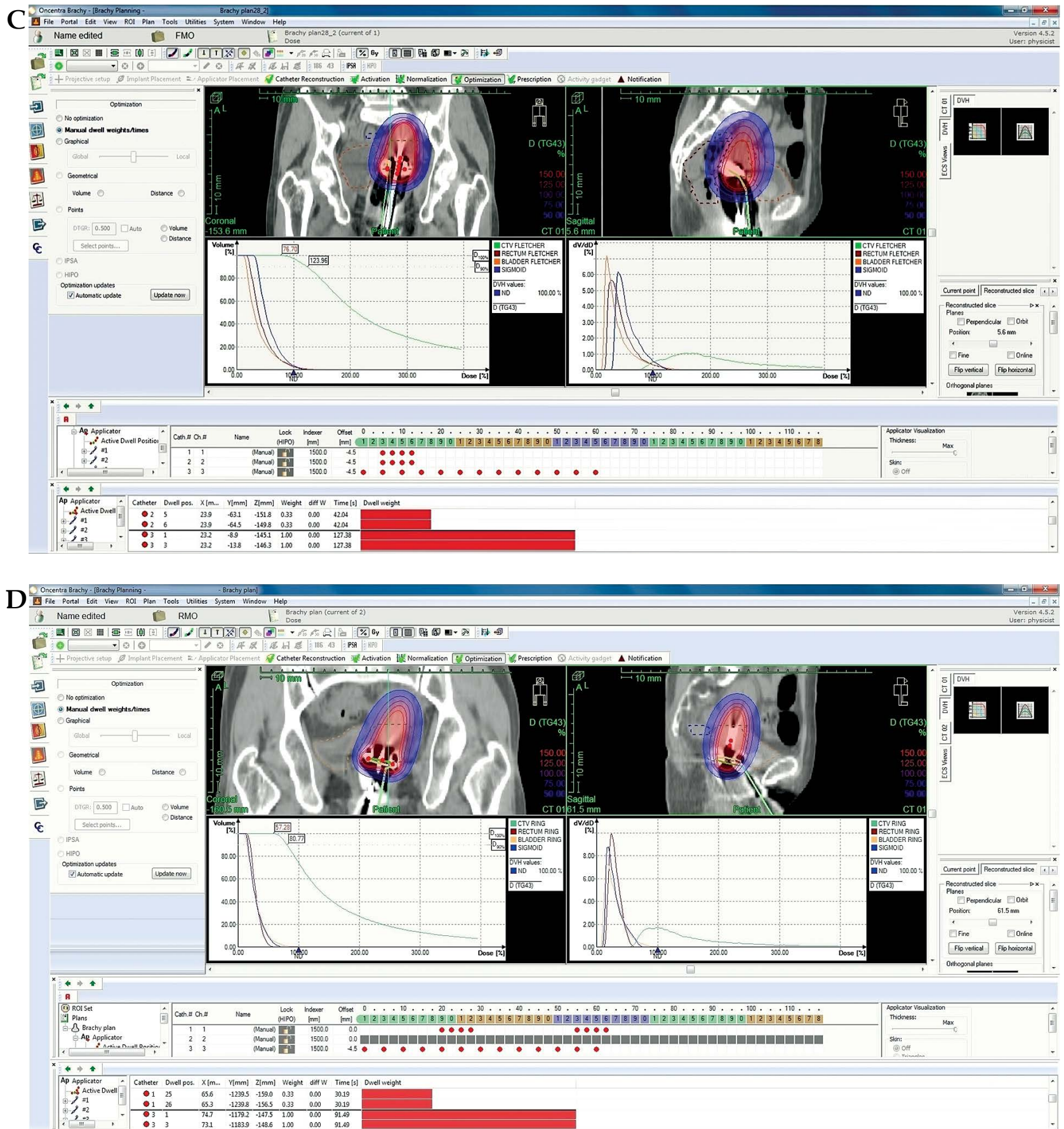

Fig. 2. Cont. C) Screenshots showing loading patterns, optimization techniques, isodose volumes, and cumulative and differential dose volume histogram generated after 'manual dwell weights/time' optimization using Fletcher tandem ovoid applicators. D) Although the loading patterns and optimization techniques are similar to Fletcher applicator as in Figure 2C, the 100\% isodose is narrower and thinner, and dose delivered to contoured volumes are lower after using ring applicator 

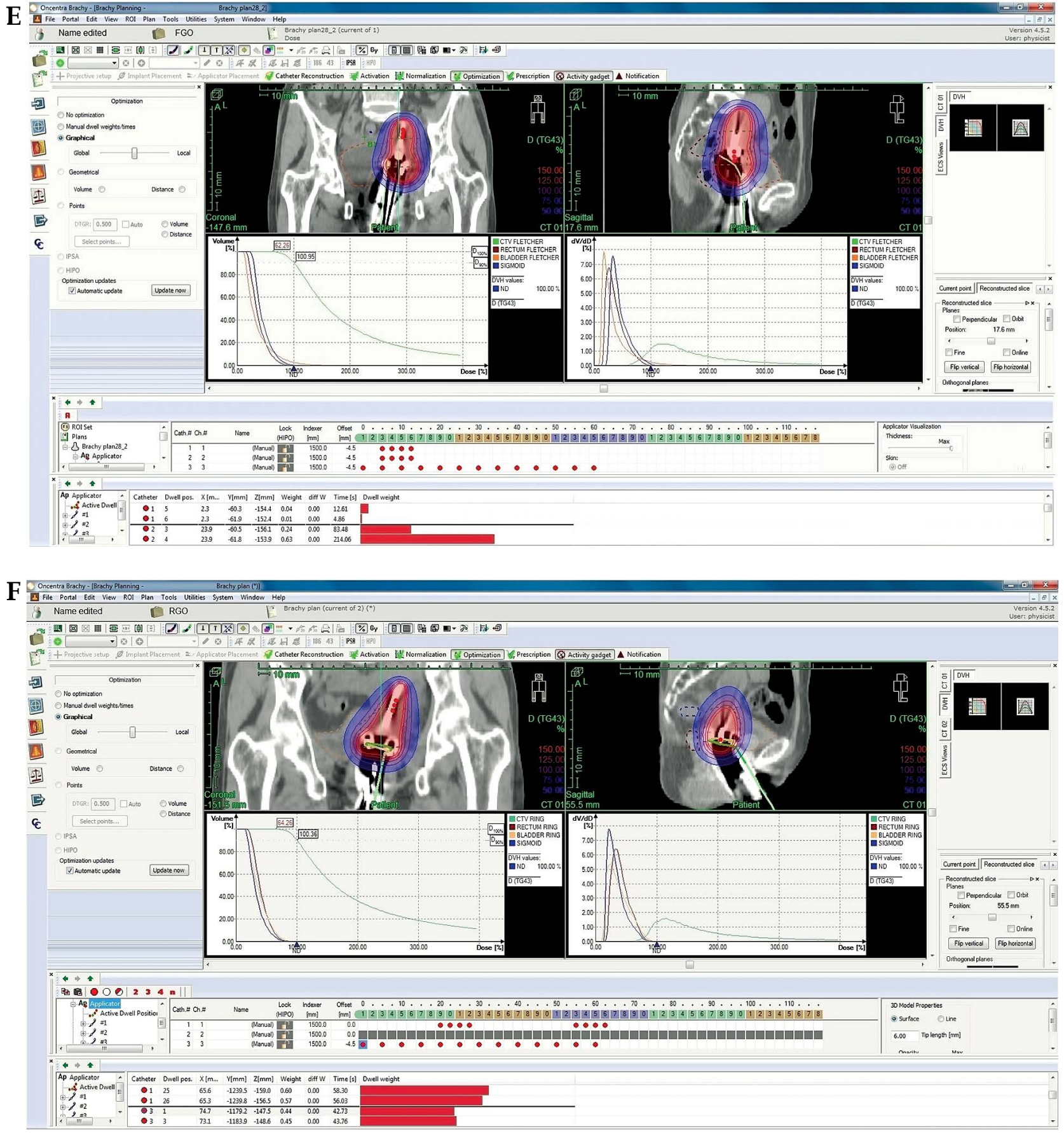

Fig. 2. Cont. E) Screenshots showing loading patterns, optimization techniques, isodose volumes, and cumulative and differential dose volume histogram generated in after 'graphical optimization' using Fletcher tandem ovoid applicators. F) The loading patterns and optimization techniques after using ring applicator were similar to Fletcher applicator as in Figure 2E. Since graphical optimization was done to achieve same dose-volume objectives, width and index thickness of $100 \%$ isodose is similar. Comparison of dose volume parameters, however, reveals (Table 2) both adequate coverage of target and sparing of organs at risk is possible after graphical optimization with ring applicators in all high-dose-rate fractionation schedules

right side), both point $B$ (Table 3), minimum dose to CTV (Table 2), and treatment time remain significantly higher with the use of Fletcher applicator. Recorded maximum dose to CTV is very high and the same for both appli- cators, and in all plans as it is located in the immediate vicinity of the applicators. Maximum height of $100 \%$ isodose curve is not statistically different due to identical loading patterns (Table 3). 


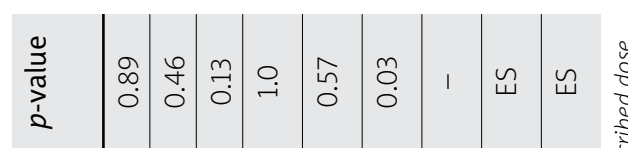

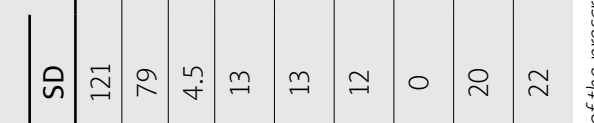

잃

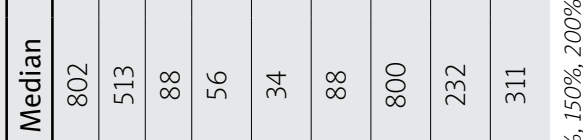

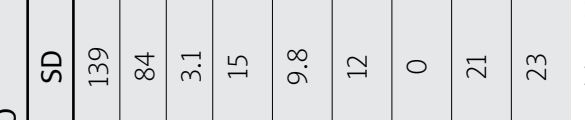

언

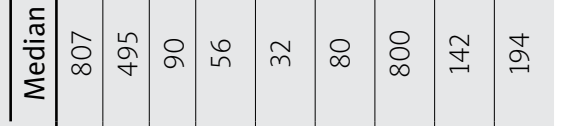

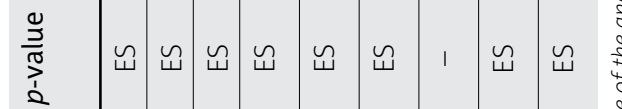

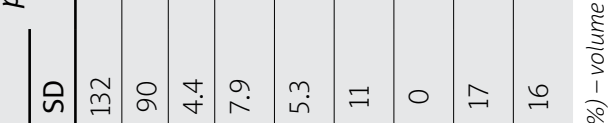

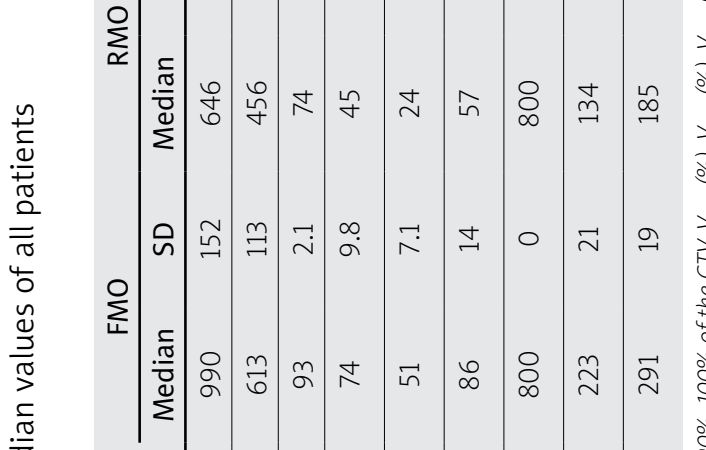

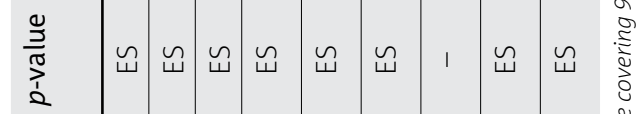
ดิ $\sum_{x}^{\circ}$

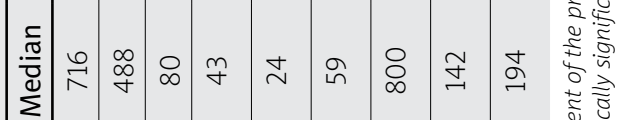

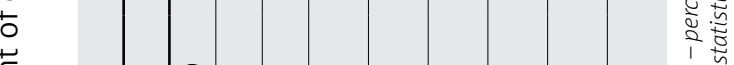

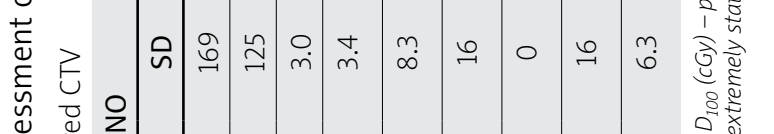

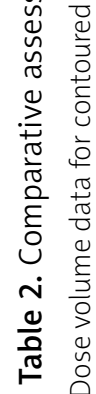

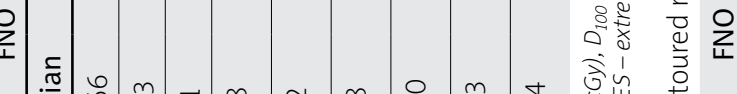

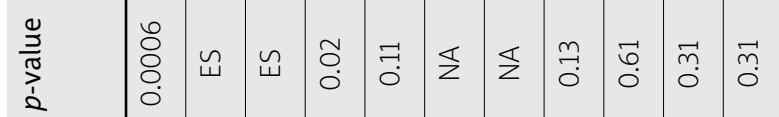

응

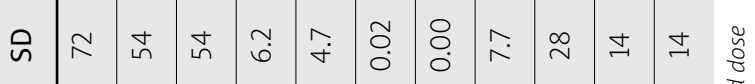

.

언

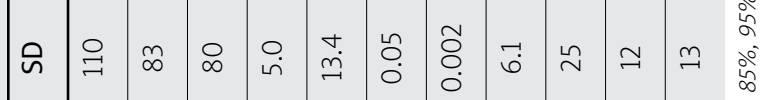

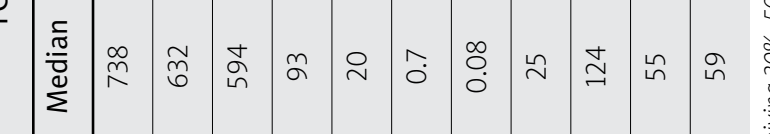

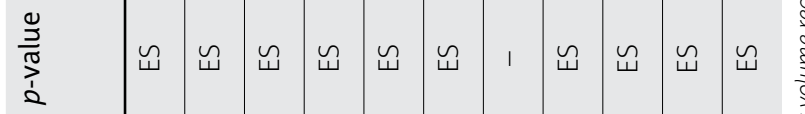

$\sum_{\propto}^{\circ}$

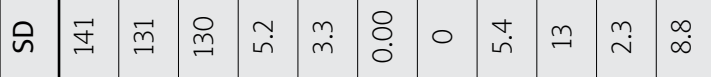

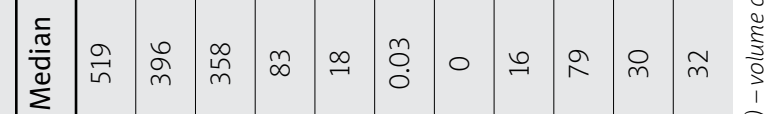

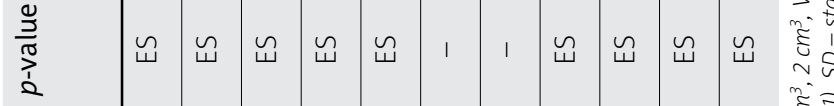

$\sum_{\propto}^{\circ}$

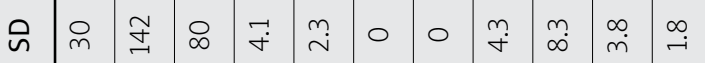

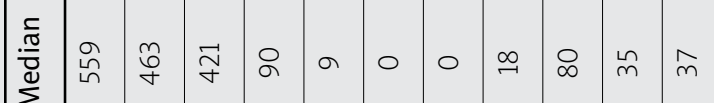

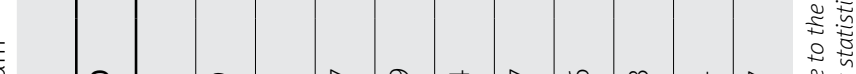

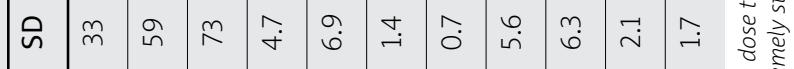

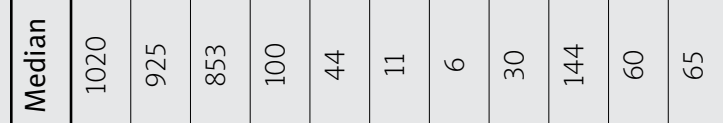

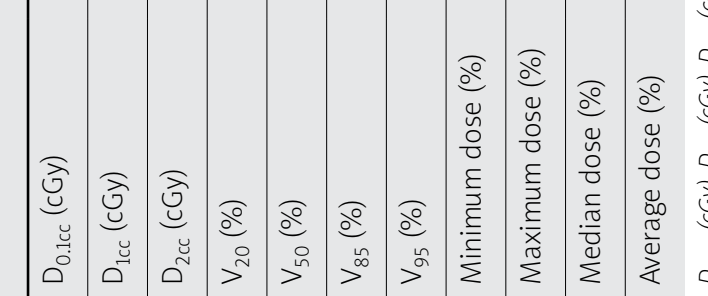




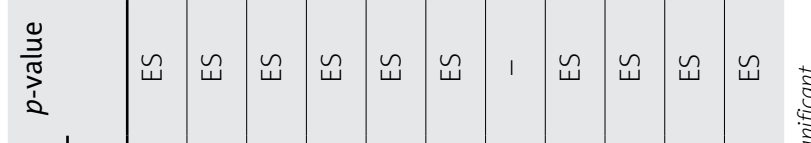

웅 ด

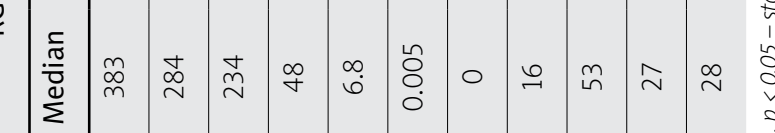

언

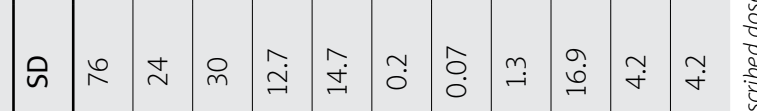

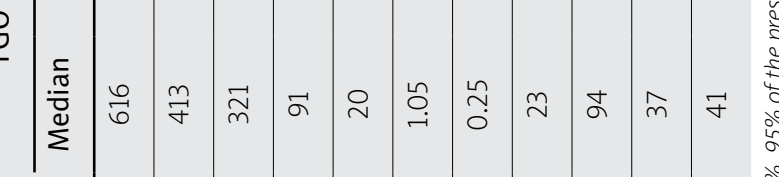

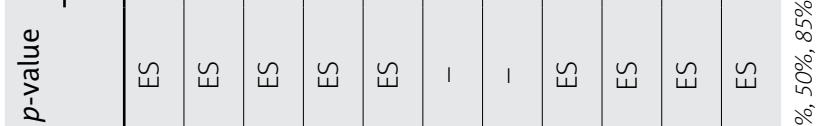

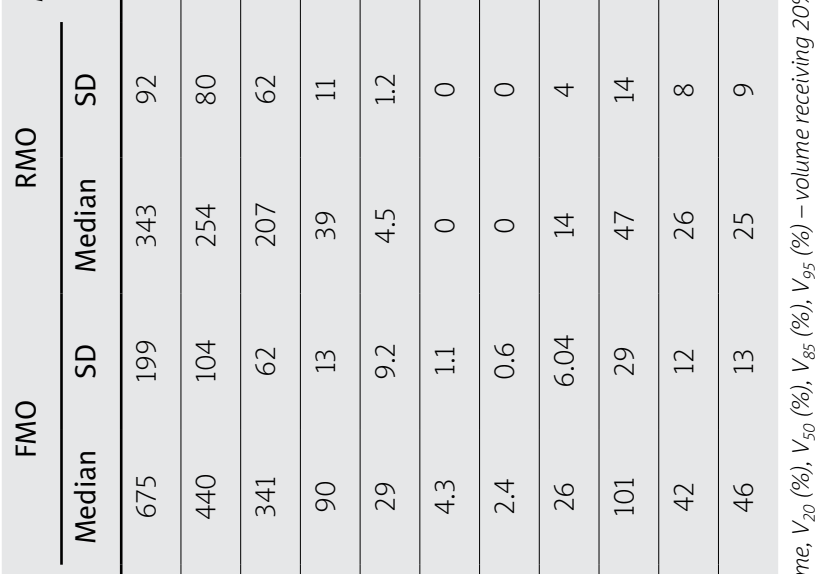

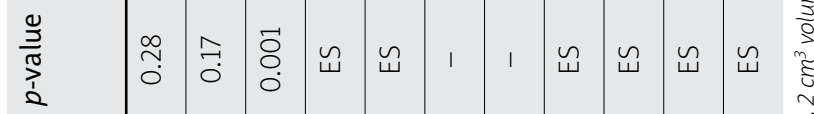

$\sum_{\propto}$

흐

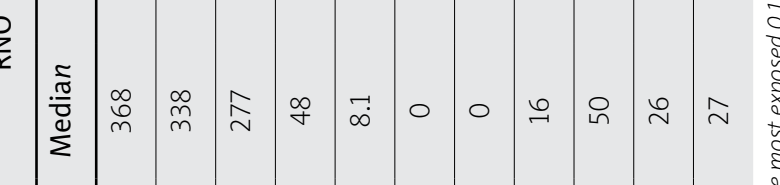

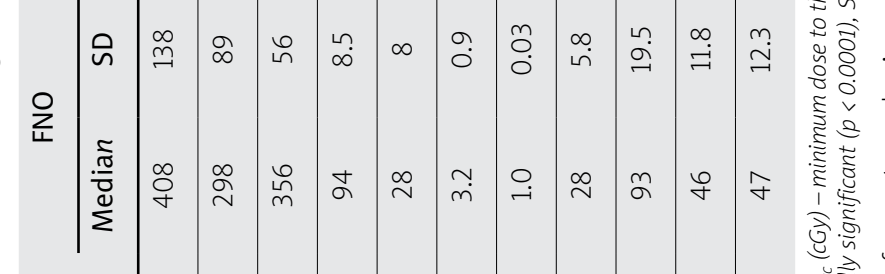

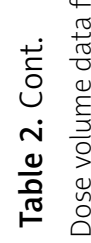




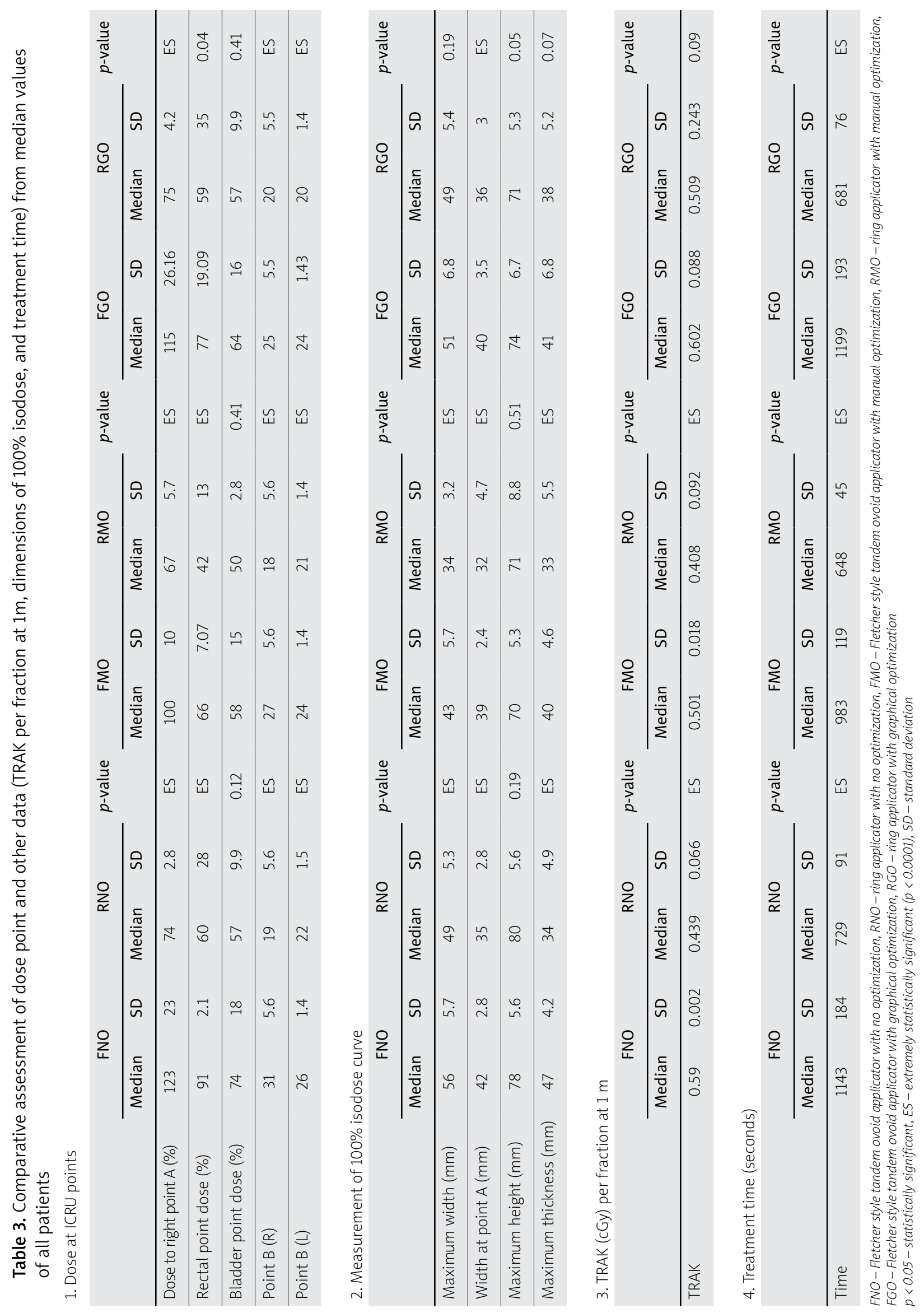



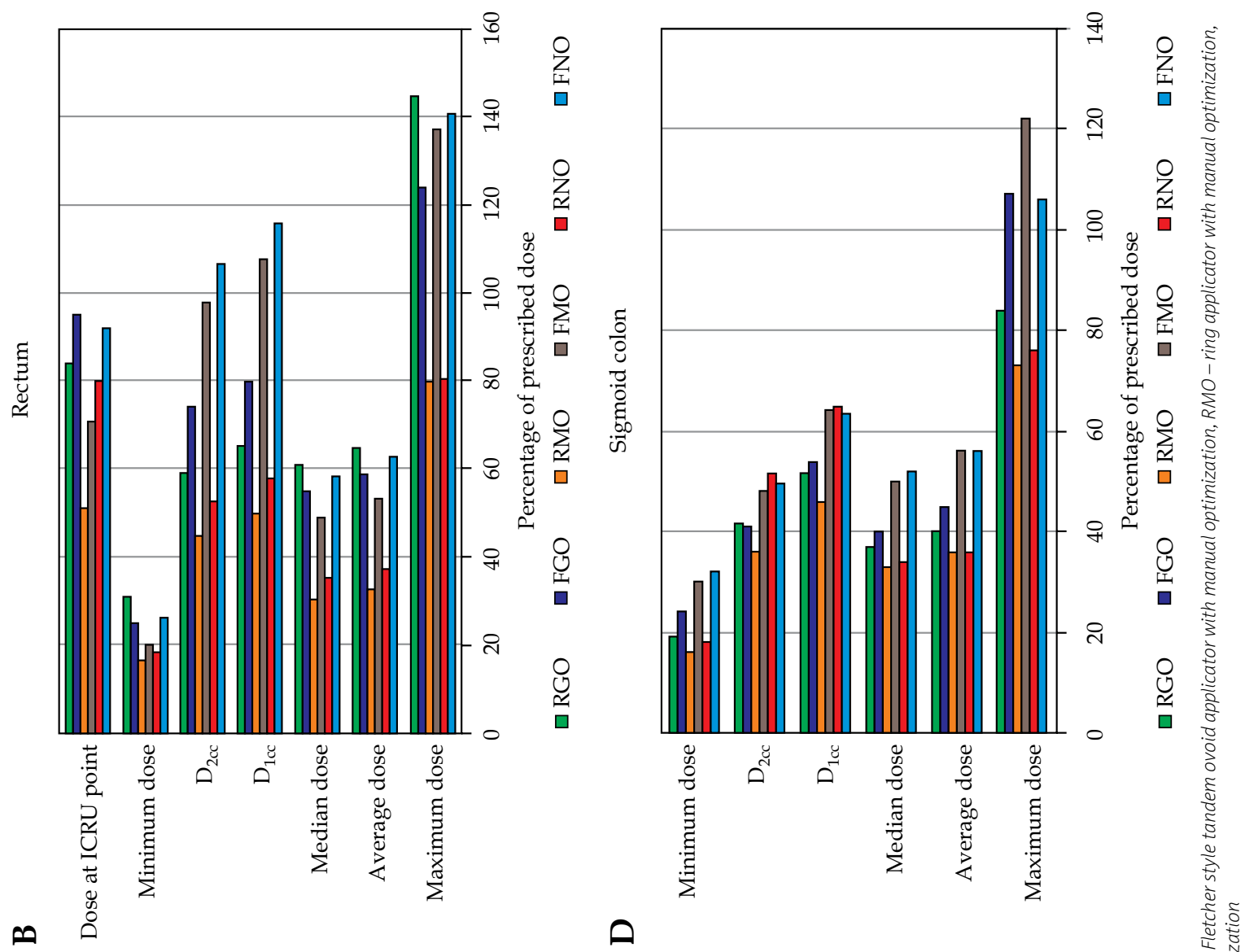

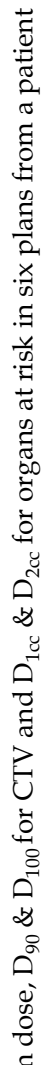

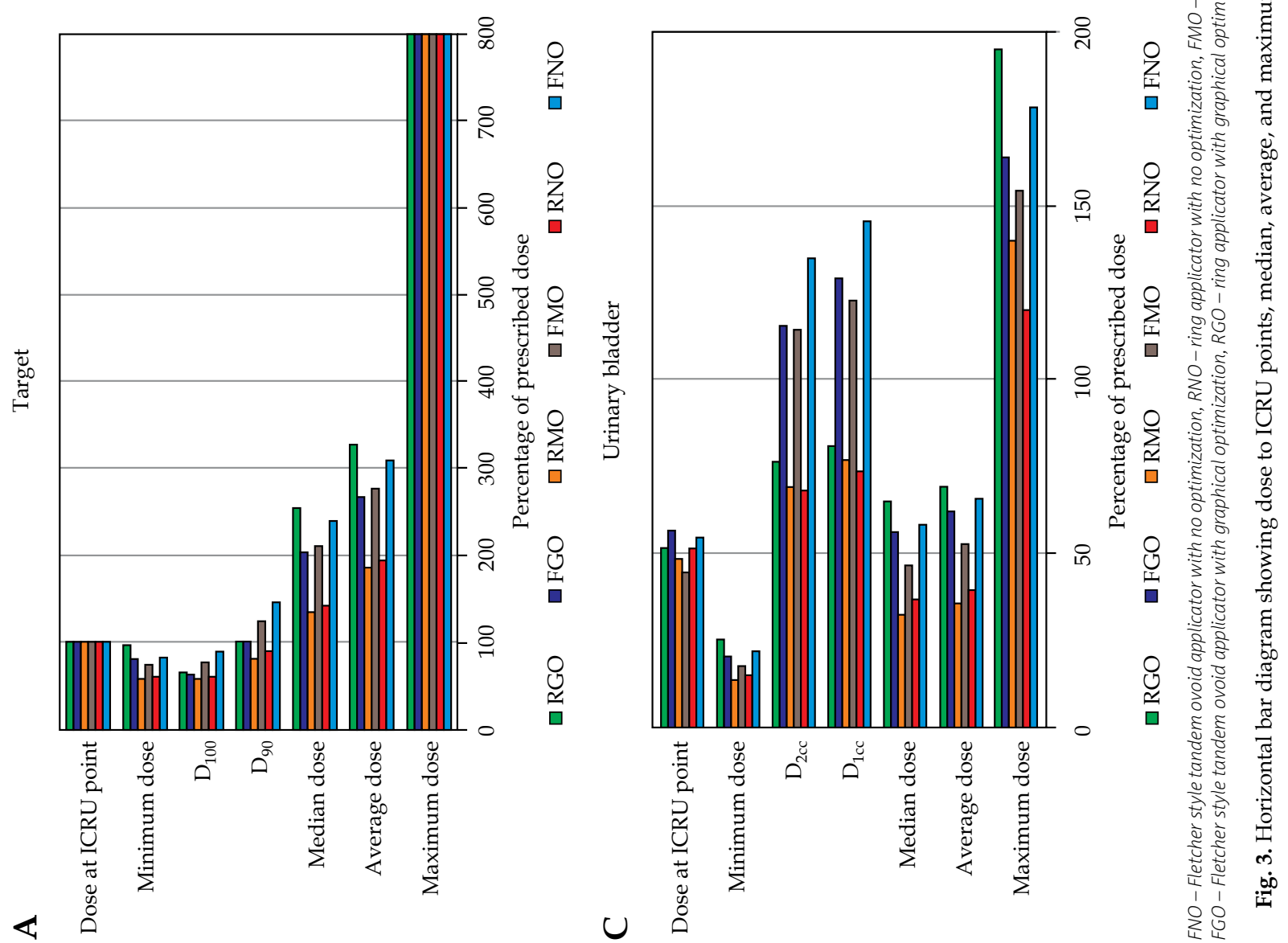




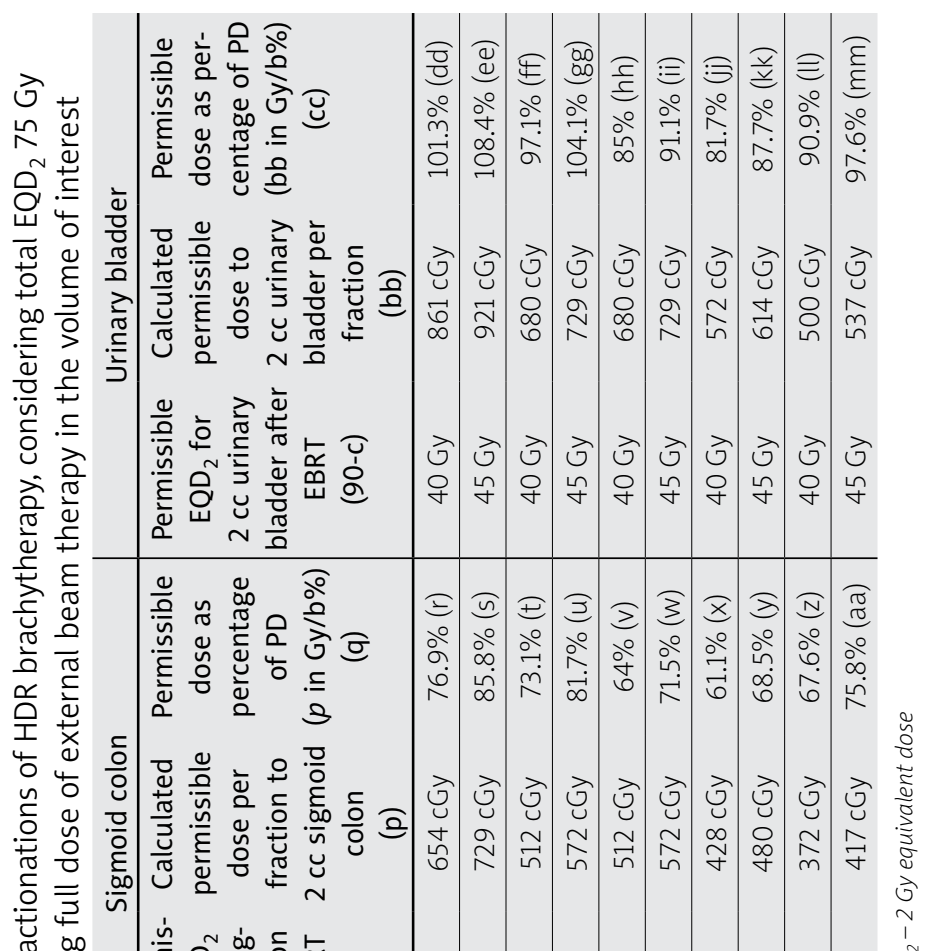

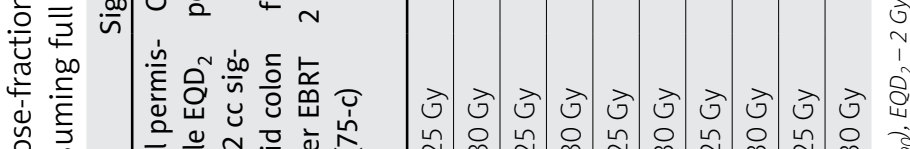

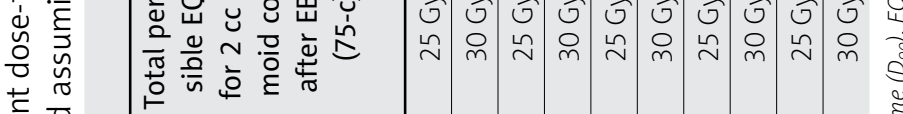

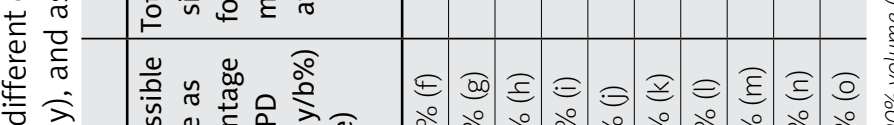

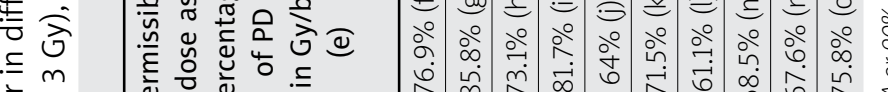

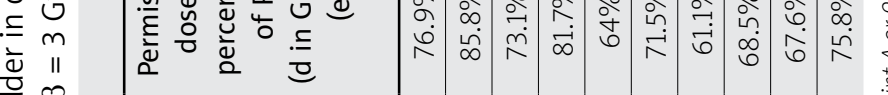

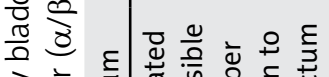

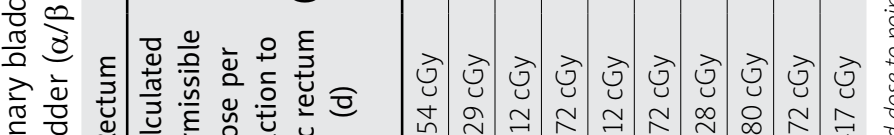

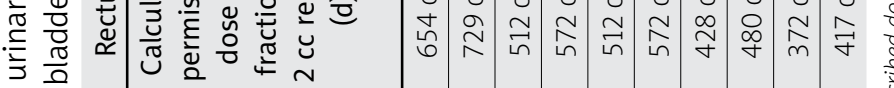

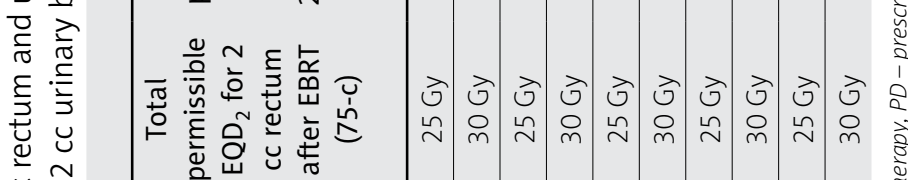

¿

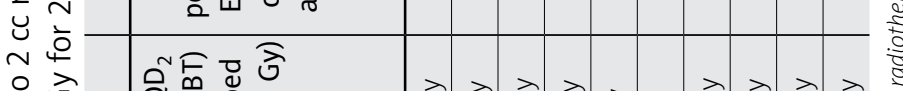

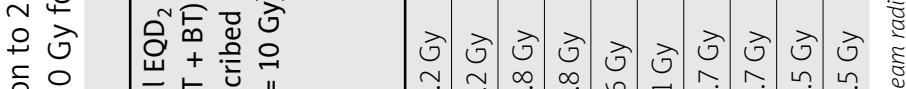

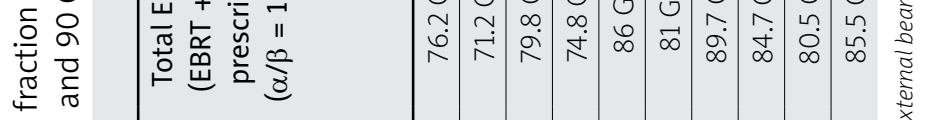

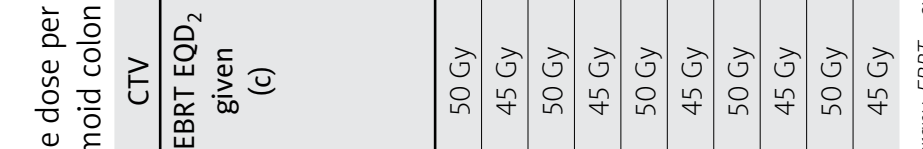

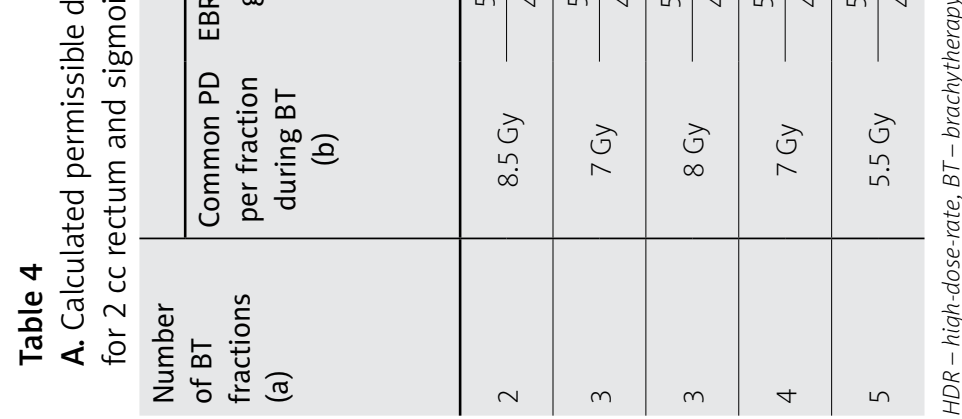

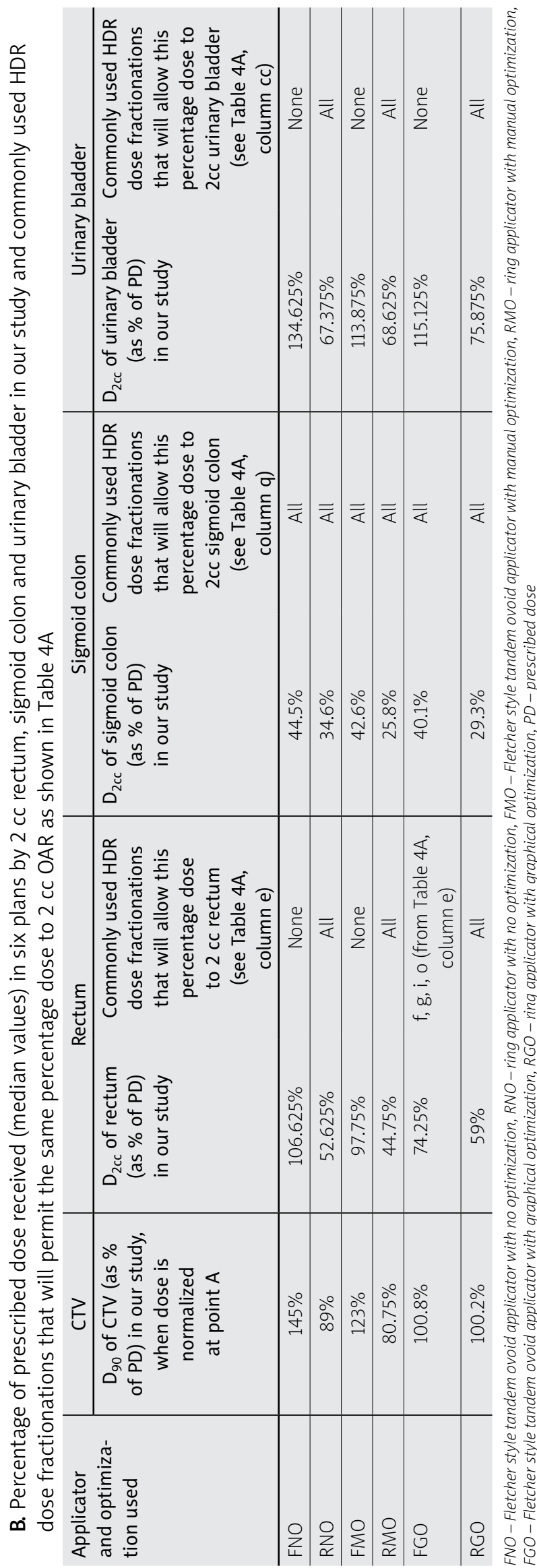




\section{Dose to rectum}

Since Fletcher applicator delivers dose both wider and thicker, $\mathrm{D}_{0.1 \mathrm{cc},}, \mathrm{D}_{1 \mathrm{cc},} \mathrm{D}_{2 \mathrm{cc}}$ and $\mathrm{V}_{20}$ of rectum (Table 2) and dose to rectal point (Table 3 ) are significantly higher in all optimization techniques with Fletcher applicator. However, difference in recorded minimum, maximum, median, and average dose as well as $\mathrm{V}_{50}$ is significantly higher only if graphical optimization is not carried out.

\section{Dose to sigmoid colon}

Although $\mathrm{D}_{0.1 \mathrm{cc}}$ and $\mathrm{D}_{1 \mathrm{cc}}$ are not significantly different between two applicators when no optimization is done, Fletcher applicator renders significantly higher $\mathrm{D}_{2 \mathrm{cc}}, \mathrm{V}_{20}$, $\mathrm{V}_{50}$, minimum, maximum, median, and average dose to sigmoid colon for all optimization techniques. However, doses are actually much lesser than that received by rectum as the sigmoid lies farther from the sources.

\section{Dose to urinary bladder}

$\mathrm{D}_{0.1 \mathrm{cc}}, \mathrm{D}_{1 \mathrm{cc},}, \mathrm{D}_{2 \mathrm{cc}}$ of urinary bladder are significantly higher with Fletcher applicator. As with rectum, difference in recorded minimum, maximum, median, and average dose is significantly higher only if graphical optimization is not done (Table 2). Dose to bladder point is not significantly different $(p>0.05)$.

Interestingly, when ring applicator along with graphical optimization is used, $V_{20}$ in rectum and both $V_{20}$ and $\mathrm{V}_{50}$ in urinary bladder, becomes statistically significantly higher. The probable explanation is that when higher isodose lines are dragged away from the contoured OAR in graphical optimization, the lower isodose regions come within automatically.

\section{Which applicator/optimization is the best for satisfying both CTV and OAR dose volume recommendations at the same time?}

Figure 3 demonstrates that when treatment plans are normalized to point $\mathrm{A}$, the use of ring applicator delivers lower dose to both CTV and OAR when compared to Fletcher applicator. Furthermore, the use of optimization using 'manual dwell weights/time', when compared to no optimization, reduces dose to most of the volumes. Although rectum and urinary bladder are well protected with use of ring applicator, the dose to $90 \%$ of CTV is inadequate unless graphical optimization is performed. On the other hand, Fletcher applicator provides adequate coverage to CTV, but also stretches very high dose to the organs at risk for all optimization processes.

To investigate the best combination of applicator and optimization for different HDR fractionations, we calculated permissible dose per brachytherapy fraction to $2 \mathrm{cc}$ of rectum, sigmoid colon, and urinary bladder to keep $\mathrm{EQD}_{2}$ to these volumes within recommended limits (see Table 4A). Permissible dose per fraction was then expressed as percentage of prescribed dose in different fractionations and compared to the results obtained from our study (Table 4B). Results show that both adequate coverage to CTV $\left(D_{90}=100 \%\right.$ prescribed dose $)$ and dose restric- tion to rectum $\left(D_{2 c c}<75\right.$ Gy $\left.E Q D_{2}\right)$ is possible after use of graphical optimization with Fletcher applicator, only in selected HDR dose-fractionation schedules (8.5 Gy for 2 fractions after external irradiation with $\mathrm{EQD}_{2}$ of $45-50 \mathrm{~Gy}$, and 7 Gy for 3 fractions and 5.5 Gy for 5 fractions after EBRT with $\mathrm{EQD}_{2}$ of $45 \mathrm{~Gy}$ ). When Fletcher applicator is used, urinary bladder always receive dose higher than recommended, even if graphical optimization is used with an intent not to compromise dose to CTV. Since sigmoid colon receives lesser dose, $\mathrm{D}_{2 \mathrm{cc}}$ is within permissible range in all optimization techniques, and both applicators for all HDR fractionations. On the other hand, ring applicator provides suboptimal $\mathrm{D}_{90}$ of CTV unless plan is graphically optimized. Thus, both adequate coverage of the target and sparing of the organs at risk simultaneously is possible in all fractionation schedules, only after graphical optimization with ring applicators. Judicious activation of dwell positions and graphical optimization after the use of Fletcher applicator, however, may generate desired dose volume parameters for all volumes of interest in other HDR fractionation schedules also.

\section{Conclusions}

The choice of brachytherapy applicators depends on their availability, patient's anatomy and the decision of the radiation oncologist. The optimization technique to be adopted however depends on medical physicist's preference and the readiness to adhere to dose-volume objectives. In this study, the combination of graphical optimization and the application of ring applicators easily engendered realization of dose-volume objectives in all HDR dose-fractionation schedules. On the other hand, Fletcher style tandem-ovoid applicator generates very high dose to $2 \mathrm{cc}$ volumes of OAR in addition to high-dose to CTVs. Further studies with these two applicator systems should be conducted with larger number of patients. These may include optimization techniques like IPSA and HIPO. Rectal and bladder wall contouring may be attempted if MRI is used for imaging.

\section{Disclosure}

Authors report no conflict of interest.

\section{References}

1. Torre LA, Bray F, Siegel RL et al. Global cancer statistics, 2012. CA Cancer J Clin 2015; 65: 87-108.

2. Cervical cancer estimated incidence, mortality and prevalence worldwide in 2012. Available at: http:/ / globocan.iarc.fr/old/ FactSheets/cancers/cervix-new.asp [Accessed: 19 April 2017].

3. Viswanathan AN, Creutzberg CL, Craighead P et al. International brachytherapy practice patterns: a survey of the Gynecologic Cancer Intergroup (GCIG). Int J Radiat Oncol Biol Phys 2012; 82: 250-255.

4. Basu B, Basu S, Chakraborti B et al. A comparison of dose distribution from Manchester style and Fletcher-style intracavitary brachytherapy applicator systems in cervical cancer. J Contemp Brachyther 2012; 4: 213-218.

5. Viswanathan AN, Beriwal S, De Los Santos J et al. The American Brachytherapy Society Treatment Recommendations for Locally Advanced Carcinoma of the Cervix Part II: High Dose-Rate Brachytherapy. Brachytherapy 2012; 11: 47-52. 
6. American Brachytherapy Society cervical cancer brachytherapy task group. Viswanathan AN, Thomadsen B. Available at: https:/ / www.americanbrachytherapy.org/guidelines/cervical_cancer_taskgroup.pdf [Accessed: 19 April 2017].

7. Pötter R, Haie-Mederb C, Van Limbergenc E et al. Recommendations from gynaecological (GYN) GEC ESTRO working group (II): Concepts and terms in 3D image-based treatment planning in cervix cancer brachytherapy-3D dose volume parameters and aspects of 3D image-based anatomy, radiation physics, radiobiology. Radiother Oncol 2006; 78: 67-77.

8. Ibhade OR, Oyeyemi OE, Idayat AB et al. Tandem-ring dwell time ratio in Nigeria: dose comparisons of two loading patterns in standard high-dose-rate brachytherapy planning for cervical cancer. J Contemp Brachytherapy 2015; 7: 161-170.

9. Suneja G, Brown D, Chang A et al. American Brachytherapy Society: Brachytherapy treatment recommendations for locally advanced cervix cancer for low-income and middle-income countries. Brachytherapy 2017; 16: 85-94.

10. Yin G, Wang P, Lang J et al. Dosimetric study for cervix carcinoma treatment using intensity modulated radiation therapy (IMRT) compensation based on 3D intracavitary brachytherapy technique. J Contemp Brachytherapy 2016; 8: 221-232.

11. Tan PW, Koh VY, Tang JI. Outpatient combined intracavitary and interstitial cervical brachytherapy: barriers and solutions to implementation of a successful programme - a single institutional experience. J Contemp Brachytherapy 2015; 7: 259-263.

12. Dimopoulos JC, Petrow P, Tanderup K et al. Recommendations from Gynaecological (GYN) GEC-ESTRO Working Group (IV): Basic principles and parameters for MR imaging within the frame of image based adaptive cervix cancer brachytherapy. Radiother Oncol 2012; 103: 113-122.

13. Lindegaard JC, Fokdal LU, Nielsen SK et al. MRI-guided adaptive radiotherapy in locally advanced cervical cancer from a Nordic perspective. Acta Oncol 2013; 52: 1510-1519.

14. Dimopoulos JC, Schard G, Berger D et al. Systematic evaluation of MRI findings in different stages of treatment of cervical cancer: potential of MRI on delineation of target, pathoanatomic structures, and organs at risk. Int J Radiat Oncol Biol Phys 2006; 64: 1380-1388.

15. Srivastava A, Datta NR. Brachytherapy in cancer cervix: Time to move ahead from point A? World J Clin Oncol 2014; 5: 764-774.

16. Dolezel M, Odrazka K, Zizka J et al. MRI-based preplanning using CT and MRI data fusion in patients with cervical cancer treated with 3D-based brachytherapy: feasibility and accuracy study. Int J Radiat Oncol Biol Phys 2012; 84: 146-152.

17. Viswanathan AN, Dimopoulos J, Kirisits C et al. Computed tomography versus magnetic resonance imaging-based contouring in cervical cancer brachytherapy: results of a prospective trial and preliminary guidelines for standardized contours. Int J Radiat Oncol Biol Phys 2007; 68: 491-498.

18. Datta NR, Srivastava A, Maria Das KJ et al. Comparative assessment of doses to tumor, rectum, and bladder as evaluated by orthogonal radiographs vs. computer enhanced computed tomography-based intracavitary brachytherapy in cervical cancer. Brachytherapy 2006; 5: 223-229.

19. Goyal MK, Rai DV, Kehwar TS et al. Anatomy-based definition of point A utilizing three-dimensional volumetric imaging approach for high-dose-rate (HDR) intracavitary brachytherapy dose prescription when treating cervical cancer using limited resources. J Appl Clin Med Phys 2016; 17: 69-77.

20. Wachter-Gerstner N, Wachter S, Reinstadler E et al. Bladder and rectum dose defined from MRI based treatment planning for cervix cancer brachytherapy: comparison of dose-volume histograms for organ contours and organ wall, comparison with ICRU rectum and bladder reference point. Radiother Oncol 2003; 68: 269-276.
21. Siavashpour Z, Aghamiri MR, Jaberi R et al. Optimum organ volume ranges for organs at risk dose in cervical cancer intracavitary brachytherapy. J Contemp Brachytherapy 2016; 8: 135-142.

22. Patra NB, Manir KS, Basu $S$ et al. Effect of bladder distension on dosimetry of organs at risk in computer tomography based planning of high-dose-rate intracavitary brachytherapy for cervical cancer. J Contemp Brachytherapy 2013; 5: 3-9.

23. EMBRACE Study Committee. EMBRACE: A European Study on MRI-guided brachytherapy in locally advanced cervical cancer. https://www.embracestudy.dk/AboutProtocolDownload.aspx [Accessed: July 2017].

24. Cancer Care Ontario; Imaging Strategies for Definitive Intracavitary Brachytherapy of Cervical Cancer. 2014. https:// www.cancercare.on.ca/common/pages/UserFile.aspx?file-Id=309545 [Accessed: July 2017].

25. Datta NR, Basu R, Das KJ et al. Problems in reporting doses and volumes during multiple high-dose-rate intracavitary brachytherapy for carcinoma cervix as per ICRU Report 38: a comparative study using flexible and rigid applicators. Gynecol Oncol 2003; 91: 285-292.

26. Rehman AU, Buzdar SA, Hassan M et al. Impact of brachytherapy applicators geometry on dose distribution in gynaecological cancer. J Pak Med Assoc 2016; 66: 1566-1570.

27. Levin D, Menhel J, Rabin T et al. Dosimetric comparison of tandem and ovoids vs. tandem and ring for intracavitary gynecologic applications. Med Dosim 2008; 33: 315-320.

28. Ma JK, Mourad WF, Allbright R et al. Short-term clinical outcome and dosimetric comparison of tandem and ring versus tandem and ovoids intracavitary applicators. J Contemp Brachytherapy 2015; 7: 218-223. 